\title{
Besser behandeln mit Fortbildung, Kommunikation und Checklisten
}

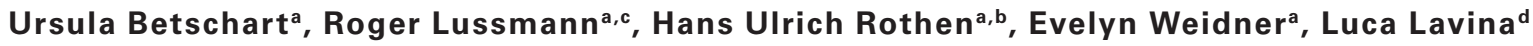 \\ ${ }^{a}$ Mitglieder der Schweizerischen Gesellschaft für Intensivmedizin (SGI); ${ }^{b}$ Prof. Dr. med.; ${ }^{c}$ Dr. med.; ${ }^{d}$ SGI-Generalsekretariat
}

Im vergangenen Herbst organisierte die Schweizerische Gesellschaft für Intensivmedizin (SGI) zum fünften Mal das interdisziplinäre und interprofessionelle ICU Management Seminar. Am Vortag der gemeinsamen Jahrestagung der SGI mit den Schweizerischen Gesellschaften für Infektiologie (SGInf), Spitalhygiene (SGSH), Tropenmedizin und Parasitologie (SGTP) sowie Tropen- und Reisemedizin (SGTRM) widmete sich diese Veranstaltung verschiedenen Themen, welche für die Patientensicherheit in zahlreichen medizinischen Fachgebieten von Belang sind.

\section{Strukturierte und sichere Patienten- übergaben}

In allen Disziplinen, die stationär Patientinnen und Patienten behandeln, stellt deren Übergabe an das Behandlungsteam einer nächsten Schicht oder einer anderen Abteilung ein gewisses Risiko für die Sicherheit der Patientinnen und Patienten dar. Schliesslich beeinträchtigen etwa unvollständige oder inkorrekte Informationen die Qualität von Entscheidungen und somit auch der Behandlung. Wie Nikolai Hulde vom Herz- und Diabeteszentrum in Bad Oeynhausen, Deutschland, erwähnt, kann sich die Medizin zur Verbesserung der Kommunikation bei Übergaben etwa eines Konzepts bedienen, das ursprünglich von der amerikanischen Marine für die sichere und effiziente Kommunikation in Atom-U-Booten entwickelt wurde. Mit Hilfe dieses sogenannten SBAR-Modells, ein Akronym aus den Bezeichnungen der vier einzelnen Schritte des Modells (Situation, Background, Assessment und Recommendation), werden in einer genau definierten Abfolge Informationen über die Patientin oder den Patienten und ihre oder seine Gesundheitssituation präsentiert. Dadurch wird das Übergabeprotokoll strukturiert, Missverständnisse werden vermieden und sowohl das Teamwork wie auch die Patientensicherheit werden verbessert. Nach einer Bereitstellung von persönlichen Patientenangaben wie Namen, Alter, Geschlecht und Informationen zu aktuellen Vitalzeichen (Situation) werden Hintergrundinformationen präsentiert, die für die Behandlung des Patienten oder der Patientin zentral sind (Background). Dazu ge- hören etwa die Anamnese, Befunde von körperlichen Untersuchungen, Allergien, Komorbiditäten, aber auch nichtmedizinische Informationen, zum Beispiel zu Wertgegenständen, welche die Patientin oder der Patient beim Eintritt ins Spital bei sich hatte. In einem dritten Schritt werden die aktuelle Situation und die Arbeitsdiagnose dargelegt (Assessment), bevor die Übergabe mit einer Empfehlung zu weiteren Behandlungsmassnahmen beendet wird (Recommendation). Die genauen Bestandteile der einzelnen Phasen können zwischen unterschiedlichen medizinischen Disziplinen, Spitälern und Stationen variieren, so Olivier Pantet von der Erwachsenen-Intensivstation am Universitätsspital Lausanne (CHUV). Die von den folgenden vier Fragen geprägte Struktur ist jedoch allen Varianten des SBAR-Modells gemeinsam: Was ist passiert? (Situation), wie lautet die Vorgeschichte? (Background), was ist das Problem? (Assessment), und was sollte unternommen werden? (Recommendation).

Johannes Wacker von der Klinik Hirslanden in Zürich betont, dass es bei der Einführung des SBAR-Konzepts und der Anpassung der vier einzelnen Schritte an die Anforderungen der eigenen Disziplin und Abteilung von grosser Bedeutung ist, das eigene Behandlungsteam zu involvieren. Schliesslich sind es diese Personen, die das SBAR-Konzept erfolgreich in ihrem Alltag anwenden müssen.

\section{Vom Militär lernen}

Ein weiterer systematischer Ansatz, der mit Unterstützung des amerikanischen Militärs entwickelt wurde, 
um die Patientensicherheit durch effektive Kommunikations- und Teamarbeitsfähigkeiten zu verbessern, ist TeamSTEPPS (kurz für «Team Strategies and Tools to Enhance Performance and Patient Safety»). Wie Joëlle Hausser von der Notfallstation am Hôpital de Nyon in ihrer Präsentation erwähnt, zielt TeamSTEPPS darauf $\mathrm{ab}$, die Handlungen der Behandlungsteams so zu optimieren, dass diese schnell und effektiv auf alle möglichen auftretenden Situationen reagieren können. TeamSTEPPS verfügt über fünf Schlüsselprinzipien: Erstens müssen die Komponenten identifiziert werden, deren Zusammenspiel funktionieren muss, um Patientensicherheit zu garantieren. Zweitens soll eine effektive Kommunikation unter den einzelnen Mitgliedern des Behandlungsteams ermöglicht werden dabei kommt häufig das bereits erwähnte SBAR-Modell zum Einsatz. Drittens sollen Führungspersonen dazu in der Lage sein, Aufgaben klar zu kommunizieren und zu koordinieren, Teammitglieder zu motivieren, um eine optimale Leistung zu ermöglichen. Viertens sollen die Teammitglieder die aktuelle Situation erfassen und beurteilen können, um zu wissen, was um sie herum geschieht. Fünftens sollen sich die Teammitglieder in ihren Aufgaben gegenseitig aktiv unterstützen. Dabei sind ehrliche, konstruktive Rückmeldungen wichtig, auch wenn die eigene Meinung von jener der Mehrheit abweicht. Diese wichtigen Funktionen im behandelnden Team wurden auch von Thomas Sauter aus dem universitären Notfallzentrum des Berner Inselspitals hervorgehoben. Gegenseitige Schuldzuweisungen sind unproduktiv, sie schaden der Teamkultur und letztlich auch der Patientensicherheit: «Stop the culture of blame.»

\section{Mehr als nur Häkchensetzen}

Im klinischen Alltag sind häufig Checklisten im Einsatz, um sicherzustellen, dass Tätigkeiten geordnet und vollständig durchgeführt werden. Gemäss Lorin M. Benneker von der Universitätsklinik für Orthopädische Chirurgie und Traumatologie des Inselspitals und Pierre

\section{Das Wichtigste in Kürze}

- Übergaben stellen ein gewisses Risiko für die Sicherheit der jeweiligen Patientinnen und Patienten dar. Das SBAR-Modell kann diesen Prozess strukturieren und so verbessern.

- DerTeamSTEPPS-Ansatz zielt darauf ab, die Handlungen der Behandlungsteams so zu optimieren, dass diese schnell und effektiv auf alle möglichen Situationen reagieren können.

- Im klinischen Alltag werden häufig Checklisten eingesetzt, um sicherzustellen, dassTätigkeiten strukturiert und komplett durchgeführt werden.

- Instrumente zur Erhöhung der Patientensicherheit sollten unter Einbezug des Behandlungsteams implementiert und überarbeitet werden.

- Eine regelmässige Aktualisierung des Wissens und der Fähigkeiten des Behandlungsteams ist für eine hohe Patientensicherheit unerlässlich.
Chopard vom Service Qualité des Soins an den Hôpitaux Universitaires de Genève (HUG) erhöhen Checklisten nicht nur die Sicherheit medizinischer Behandlungen, sondern auch deren Effizienz. Schliesslich können mit Checklisten, die etwa im perioperativen Kontext eingesetzt werden, bevorstehende Schritte eines Eingriffs vom Operationspersonal bereits vorbereitet werden.

Anja Brunsveld-Reinders vom Leiden University Medical Center in den Niederlanden berichtet am ICU Management Seminar 2019 von ihren Erfahrungen mit der Entwicklung einer Checkliste für den spitalinternen Transport. Dieser stellt besonders bei kritisch kranken Patientinnen und Patienten, die auf aufwendige intensivmedizinische Massnahmen wie künstliche Beatmung sowie eine ausführliche Überwachung diverser Organfunktionen angewiesen sind, eine grosse organisatorische und logistische Herausforderung und entsprechend auch ein Risiko für die Patientinnen und Patienten dar. Zur Durchführung von bildgebenden und diagnostischen Verfahren sind solche Transporte häufig unvermeidbar. Wie das bereits erwähnte SBARModell oder andere Instrumente zur Erhöhung der Behandlungsqualität und -sicherheit profitieren auch Checklisten davon, wenn diese unter Miteinbezug des Behandlungsteams erarbeitet und regelmässig kritisch überprüft und bei Bedarf angepasst werden. Dadurch kann sichergestellt werden, dass die Instrumente jederzeit den Bedürfnissen des Teams sowie jenen der behandelten Patientinnen und Patienten entsprechen.

\section{Sicherstellen der Kompetenz durch stetige Fortbildung}

Doch nicht nur Instrumente wie die hier erwähnten, sondern auch das Wissen und die Fähigkeiten des Behandlungsteams sollten regelmässig evaluiert und aktualisiert werden. Deshalb ist die kontinuierliche und lebenslange Fortbildung von medizinischen Fachpersonen wie Ärztinnen, Ärzten und Pflegefachpersonen von grösster Bedeutung für eine hohe Behandlungsqualität und -sicherheit, wie Roger Lussmann, Präsident der SGI-Kommission für Weiter- und FortbildungÄrzte (KWFB-Ärzte), betont. Hier seien auch die Führungspersonen der Spitäler und einzelnen Abteilungen gefragt. Deren Aufgabe ist es, Weiter- und Fortbildung zu ermöglichen und auf diese Weise zu einer produktiven Arbeitsumgebung beizutragen, so Friedrich Meynen vom Eidgenössischen Nuklearsicherheitsinspektorat (ENSI). Die Förderung eines Arbeitsumfeldes, das qualitativ hochstehende Intensivmedizin ermöglicht, ist deshalb auch einer der Schwerpunkte im SGI Profil 2025. Dieses kann auf der SGI-Website unter www.sgi-ssmi.ch eingesehen werden. 